\title{
Development of Teaching Tool for Supporting Understanding of Tensor Decomposition Using MacMahon's Coloured Cubes
}

\author{
Naoki Yamamoto, Akio Ishida, Nobuhiro Oishi, and Jun Murakami
}

\begin{abstract}
In this paper, we developed tool to learn the calculation process of HOSVD. This tool uses newly created puzzle using some of MacMahon's coloured cubes. The puzzle is represented as the $2 \mathrm{D}$ map using the matrix unfolding required to calculate HOSVD. In order to investigate the difficulty level of the puzzle and the visibility of its map representation, the teaching tool is tried by our students and others at the Campus Open Day of our college. As a result, it was confirmed that most users could understand the map and that the puzzle was rather easy to solve. Therefore, it was thought that the users seemed to understand the concept of the matrix unfolding roughly.
\end{abstract}

Index Terms-MacMahon's coloured cubes, 3D puzzle, matrix unfolding, HOSVD, understanding support tools.

\section{INTRODUCTION}

Tensor decomposition is often widely used in big data analysis, fuzzy modeling, signal processing, image processing, image classification, biological signal analysis, text mining, social networking, and web hyperlink analysis [1]-[3]. This computation decomposes high-dimensional data represented by higher-order tensors into a product of a tensor and matrices or a sum of products of vectors. Note that in this paper the higher-order tensors have the same meaning as multidimensional arrays. We have been working on the medical data analysis using the tensor decomposition [4] and the development of teaching tools of this decomposition using 3D puzzles [5], [6]. Recent research in the latter field, we took a Rubik's cube and an Instant Insanity as 3D puzzles. However, it is very difficult to actually solve these puzzles, hence we recognized the need for easier puzzle.

Under the background as described above, we devised new 3D puzzle using some of the MacMahon's coloured cubes [7] The puzzle is used as a teaching tool to learn the principle of tensor decomposition, and focuses on a matrix unfolding and a folding (an inverse of the matrix unfolding) that appears in the calculation process of higher-order singular value decomposition (HOSVD) [8] where HOSVD is one of the tensor decomposition. In this paper, we first explain the MacMahon's coloured cubes, a devised 3D puzzle, and HOSVD algorithm. Then we describe the higher-order tensor

Manuscript received September 24, 2019; revised November 12, 2019. This work was supported in part by the JSPS KAKENHI (Grants-in Aid for Scientific Research) under Grant No. JP18K11596.

Naoki Yamamoto, Akio Ishida, Nobuhiro Oishi, and Jun Murakami are with Kumamoto College, National Institute of Technology, Kumamoto, 861-1102, Japan (e-mail: naoki@kumamoto-nct.ac.jp, ishida@kumamoto-nct.ac.jp, oishi@kumamoto-nct.ac.jp, jun@kumamoto-nct.ac.jp). representation of the puzzle and its map representation. Next we show teaching tool developed using this map representation. Finally, we describe the results of trials of using this tool for junior high school students and our college students at the Campus Open Day of our college. In addition, the analysis results on the level of difficulty of the puzzle, the visibility of the map representation, and the degree of understanding of the matrix unfolding are also shown.

\section{3D PuZZle Using MACMAhon’s COlOUREd CUBES}

MacMahon's cubes are cubes coloured on each side with six different colors, and 30 different cubes can be obtained by combining the colors on each side [7]. We selected four types of cubes from those 30 cubes and used them to create a new 3D puzzle. Fig. 1 shows a 3D puzzle composed of four types of cubes made by pasting color stickers on wooden cubes. The color scheme of each cube is shown as expanded views in Fig. 2. The colors used here are six colors, red (1), white (2), blue (3), green (4), yellow (5), and black (6), and the numbers in parentheses are color numbers. In these expanded views, the crossing part of the vertical and horizontal rows is the top face (red) of each cube.

This puzzle is a rectangular parallelepiped composed of $2 \times 2$ cubes when viewed from top face (red) of Fig. 1, and is solved by rearranging so that the colors of each side of this rectangular parallelepiped are the same color. We created this puzzle using MacMahon's coloured cubes as a puzzle that is easier to solve than the previously used Instant Insanity and Rubik's cube.

\section{HOSVD AND N-MODE MATRIX UNFOLDING}

\section{A. HOSVD Algorithm}

The higher order singular value decomposition (HOSVD) is the extension of the singular value decomposition (SVD) on a matrix and is used to decompose a higher order tensor of third order or higher, and is often used as one of the tensor decompositions [9]. This method is often applied to fuzzy modeling, image processing and classification, data analysis, data compression, and so on [3], [4], [9], [10]. The higher order tensor refers to a multidimensional array, for example, first, second, and third order tensors correspond to a vector, a matrix, and a three-dimensional array, respectively.

As an example of HOSVD, Fig. 3 shows a pattern diagram of decomposition in the case of the third order tensor. The original tensor $\mathcal{A}$ is decomposed into an $n$-mode product of 
one core tensor $\mathscr{B}$ and three orthogonal matrices $\boldsymbol{U}^{(n)}$, ( $n=1,2,3)$, where the $n$-mode product means a product of a tensor and a matrix, as an example of the operator, an 1-mode product is represented as $x_{1}$, and the core tensor corresponds to a diagonal matrix of SVD. Now, a HOSVD algorithm generalized to $M$-th order tensor is shown as follows:

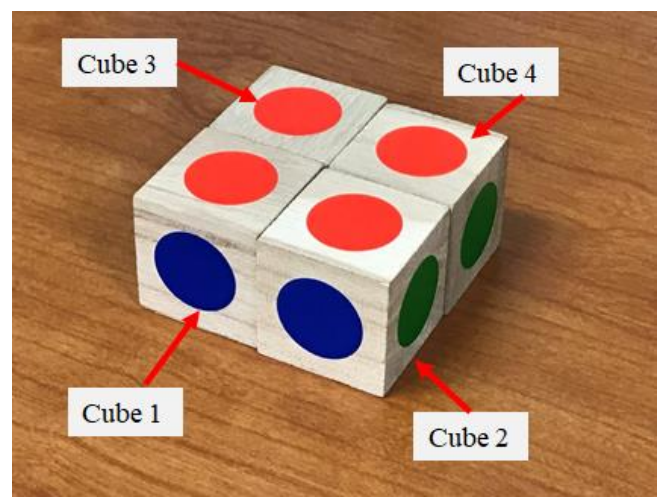

Fig. 1. Overall view of self-made 3D puzzle.

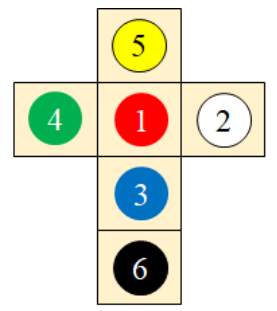

Cube 1

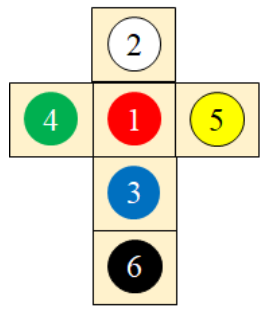

Cube 3

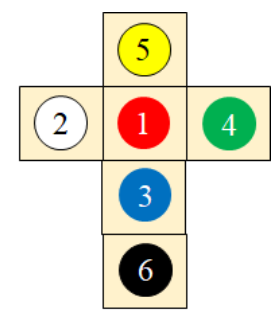

Cube 2

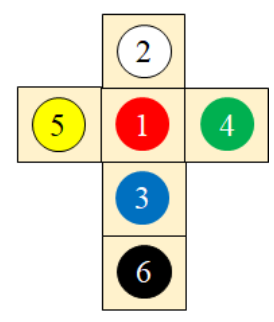

Cube 4
Fig. 2. Color scheme of each cube.

[ (Algorithm 1) HOSVD of $M$-th order tensor ]

Input: An $M$-th order tensor $\mathcal{A}$.

Output: $M$ orthogonal matrices $\boldsymbol{U}^{(n)},(n=1,2, \cdots, M)$ and a core tensor $\boldsymbol{B}$.

(Step 1) Apply the $n$-mode matrix unfolding to the $M$-th order tensor $\mathcal{A}$ to obtain $M$ types of matrices $\boldsymbol{A}_{(n)},(n=1,2, \cdots, M)$.

(Step 2) Apply SVD to the matrices $\boldsymbol{A}_{(n)}$ obtained in Step 1, respectively, to calculate $M$ left singular matrices (i.e. orthogonal matrices) $\boldsymbol{U}^{(n)},(n=1,2, \cdots, M)$.

(Step 3) Calculate the core tensor $\mathcal{B}$ by the following equation,

$$
\mathfrak{B}=\mathcal{A} \times{ }_{1} U^{(1) \mathrm{T}} \times{ }_{2} U^{(2) \mathrm{T}} \cdots \times{ }_{\mathrm{M}} U^{(\mathrm{M}) \mathrm{T}} .
$$

(Step 4) Return the matrices $\boldsymbol{U}^{(n)},(n=1,2, \cdots, M)$ and the core tensor $\boldsymbol{B}$.
(End of Algorithm)

\section{B. Definition and Algorithm for n-Mode Matrix Unfolding}

The definition of the $n$-mode matrix unfolding in Step 1 of the HOSVD algorithm described in the previous section is as follows [8]:

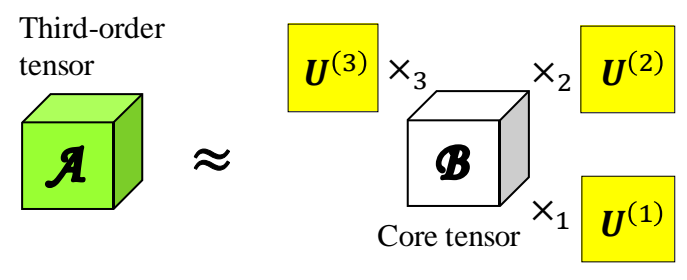

$$
\boldsymbol{A} \approx \boldsymbol{B} \times{ }_{1} \boldsymbol{U}^{(1)} \times_{2} \boldsymbol{U}^{(2)} \times_{3} \boldsymbol{U}^{(3)}
$$

Fig. 3. HOSVD of third-order tensor.
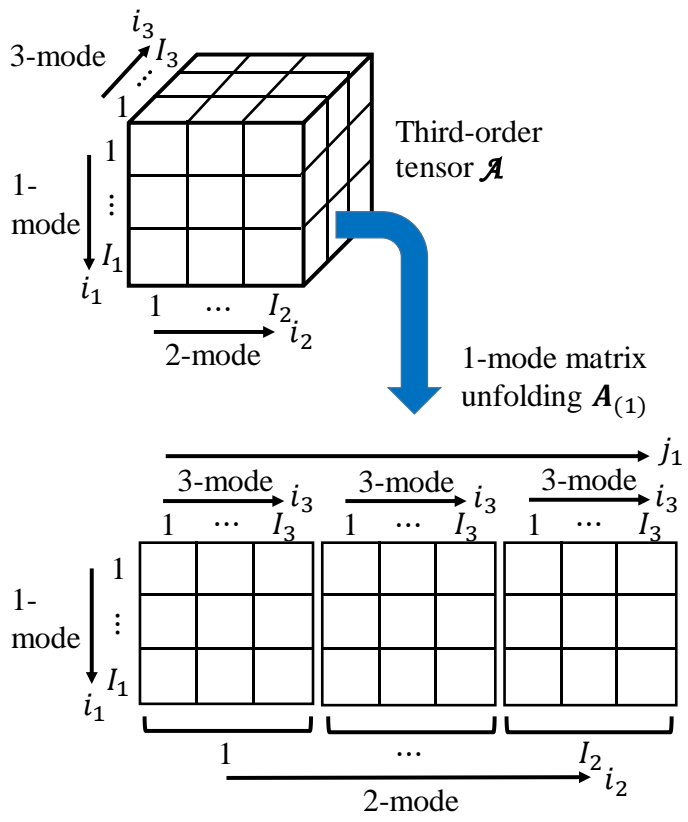

Fig. 4. 1-mode matrix unfolding of third-order tensor.

[ (Definition 1) $n$-mode matrix unfolding ]

The $n$-mode matrix unfolding is to obtain a matrix $\boldsymbol{A}_{(n)}$ of size $I_{n} \times\left(I_{n+1} I_{n+2} \cdots I_{M} I_{1} I_{2} \cdots I_{n-1}\right)$ with an $\left(i_{1}, i_{2}, \cdots, i_{M}\right)$ th element $a_{i_{1} i_{2} \cdots i_{M}}$ of the $M$-th order tensor $\mathcal{A}$ of size $I_{1} \times I_{2} \times \cdots \times I_{M}$ as an $\left(i_{n}, j_{n}\right)$ th element of $\boldsymbol{A}_{(n)}$, where the column element $j_{n}$ is given by the following formula:

$$
\begin{aligned}
j_{n}= & \left(i_{n+1}-1\right) I_{n+2} I_{n+3} \cdots I_{M} I_{1} I_{2} \cdots I_{n-1} \\
& +\left(i_{n+2}-1\right) I_{n+3} I_{n+4} \cdots I_{M} I_{1} I_{2} \cdots I_{n-1}+\cdots \\
& +\left(i_{M}-1\right) I_{1} I_{2} \cdots I_{n-1}+\left(i_{1}-1\right) I_{2} I_{3} \cdots I_{n-1} \\
& +\left(i_{2}-1\right) I_{3} I_{4} \cdots I_{n-1}+\cdots+i_{n-1} .
\end{aligned}
$$

(End of Definition)

In short, the $n$-mode matrix unfolding is an operation of converting the $M$-th order tensor into the matrix. Regarding the mode of the higher order tensor, it refers to the direction of the tensor, and in the case of the third order tensor $\mathcal{A}$ in the top of Fig. 4, the vertical, horizontal, and depth directions 
of $\mathcal{A}$ show the 1-, 2-, and 3-modes of the tensor, severally. Assuming that an $\left(i_{1}, i_{2}, i_{3}\right)$ th element of $\mathcal{A}$ is $a_{i_{1} i_{2} i_{3}}$, the corresponding relationship between the subscripts and the tensor modes represents that $i_{1}, i_{2}$, and $i_{3}$ are 1-, 2-, and 3 -modes, respectively.

TABLE I: COLOR INFORMATION OF EACH CUBE IN FIG

\begin{tabular}{|c|c|c|c|c|c|}
\hline Cube no. & Element no. & Color (Value) & Cube no. & Element no. & Color (Value) \\
\hline \multirow{6}{*}{1} & $(2,1,2,1,1)$ & Green (4) & \multirow{6}{*}{3} & $(2,1,2,1,2)$ & Green (4) \\
\hline & $(1,2,2,1,1)$ & Red (1) & & $(1,2,2,1,2)$ & Red (1) \\
\hline & $(2,2,1,1,1)$ & Blue (3) & & $(2,2,1,1,2)$ & Blue (3) \\
\hline & $(3,2,2,1,1)$ & Black (6) & & $(3,2,2,1,2)$ & Black (6) \\
\hline & $(2,2,3,1,1)$ & Yellow (5) & & $(2,2,3,1,2)$ & White (2) \\
\hline & $(2,3,2,1,1)$ & White (2) & & $(2,3,2,1,2)$ & Yellow (5) \\
\hline \multirow{6}{*}{2} & $(2,1,2,2,1)$ & White (2) & \multirow{6}{*}{4} & $(2,1,2,2,2)$ & Yellow (5) \\
\hline & $(1,2,2,2,1)$ & Red (1) & & $(1,2,2,2,2)$ & Red (1) \\
\hline & $(2,2,1,2,1)$ & Blue (3) & & $(2,2,1,2,2)$ & Blue (3) \\
\hline & $(3,2,2,2,1)$ & Black (6) & & $(3,2,2,2,2)$ & Black (6) \\
\hline & $(2,2,3,2,1)$ & Yellow (5) & & $(2,2,3,2,2)$ & White (2) \\
\hline & $(2,3,2,2,1)$ & Green (4) & & $(2,3,2,2,2)$ & Green (4) \\
\hline
\end{tabular}

According to the Definition 1, in the 1-mode matrix unfolding of $\mathcal{A}$ of Fig. 4 , since the $\left(i_{1}, i_{2}, i_{3}\right)$ th element $a_{i_{1} i_{2} i_{3}}$ of $\mathcal{A}$ is arranged in the $i_{1}$ row and the $j_{1}=\left(i_{2}-1\right) I_{3}+i_{3}$ column of the matrix $\boldsymbol{A}_{(1)}$, the whole matrix unfolding can be obtained by arranging the elements for all cases of $i_{1}=1, \cdots, I_{1}, i_{2}=1, \cdots, I_{2}, i_{3}=1, \cdots, I_{3}$ as shown in the bottom of Fig.4. Matrix unfoldings of other modes can also be obtained in the same way.

In order to obtain the matrix unfolding, it is a steady way to move each element of the higher order tensor one by one to each element of the matrix unfolding according to the Definition 1, however there is a more efficient way to implement it. That is, in the case of Fig.4, the 1-mode matrix unfolding is calculated by slicing $\mathcal{A}$ from left to right along the 2-mode of $\mathcal{A}$ and horizontally arranging the obtained matrices from left to right. An algorithm for the efficient matrix unfolding is shown below.

[ (Algorithm 2) 1-mode matrix unfolding of third order tensor ]

Input: A third order tensor $\mathcal{A}$ of size $I_{1} \times I_{2} \times I_{3}$.

Output: An 1-mode matrix unfolding $\boldsymbol{A}_{(1)}$ of size $I_{1} \times I_{2} \cdot I_{3}$.

(Step 1) Extract the submatrix $\boldsymbol{A}_{i_{2}}=\left(a_{* i_{2} *}\right)$, $\left(i_{2}=1, \cdots, I_{2}\right)$ from $\mathcal{A}$ and combine them side by side to $\boldsymbol{A}_{(1)}=\left(\boldsymbol{A}_{1}\left|\boldsymbol{A}_{2}\right| \cdots \mid \boldsymbol{A}_{I_{2}}\right)$, where $\left(a_{* i_{2} *}\right)$ is the matrix with index $i_{2}$ fixed and first and third subscripts $i_{1}=1, \cdots, I_{1}$ and $i_{3}=1, \cdots, I_{3}$.

(Step 2) Return $\boldsymbol{A}_{(1)}$.

(End of Algorithm)

\section{Higher-ORdER TENSOR REPRESENTATION OF 3D PUZZLE AND ITS MAP}

\section{A. Higher-Order Tensor Representation of 3D Puzzle}

As described earlier, the higher-order tensors used in this paper are multidimensional arrays. That is, the first to third-order tensors correspond to vectors, matrices, and 3D arrays, respectively. Here, in order to represent the puzzle shown in Fig. 1 as a multidimensional array, it is modeled by a fifth-order tensor as shown in Fig. 5. And then, each $1 \times 1 \times 1$ cube in Fig. 1 is enlarged to third-order tensor with a size of $3 \times 3 \times 3$, and the color of each face of the original cube is given to the center element of each face of the third-order tensors. Since each original cube is arranged in $2 \times 2$, the size of the fifth-order tensor is $3 \times 3 \times 3 \times 2 \times 2$.

Now, let us denote this fifth-order tensor and its $\left(i_{1}, i_{2}, i_{3}, i_{4}, i_{5}\right)$ th element as $\mathcal{A}$ and $a_{i_{1} i_{2} i_{3} i_{4} i_{5}}$, respectively. Then, the tensor $\mathcal{A}$ is expressed by the following equation:

$$
\mathcal{A}=\left(\mathrm{a}_{\mathrm{i}_{1} \mathrm{i}_{2} \mathrm{i}_{3} \mathrm{i}_{4} \mathrm{i}_{5}}\right), \quad\left(\mathrm{i}_{1}, \mathrm{i}_{2}, \mathrm{i}_{3}=1,2,3 ; \mathrm{i}_{4}, \mathrm{i}_{5}=1,2\right) .
$$

Table I shows the color values given to each element of $\mathcal{A}$. Note that all values for element numbers not shown in this table are 0 . In Fig. 5, $n$-mode $(n=1,2, \cdots, 5)$ represents the directions of the tensor data indicated by the arrows of tensor data and correspond to the subscripts $i_{1}, i_{2}, i_{3}, i_{4}$ and $i_{5}$ of $\mathcal{A}$, respectively.

\section{B. Map of 3D Puzzle}

To represent this $3 \mathrm{D}$ puzzle as a $2 \mathrm{D}$ map, we apply the $n$-mode matrix unfolding used in the calculation process of HOSVD. Here we use an 1-mode matrix unfolding especially as an algorithm for creating a puzzle map. The following is the algorithm for creating a map of this 3D puzzle.

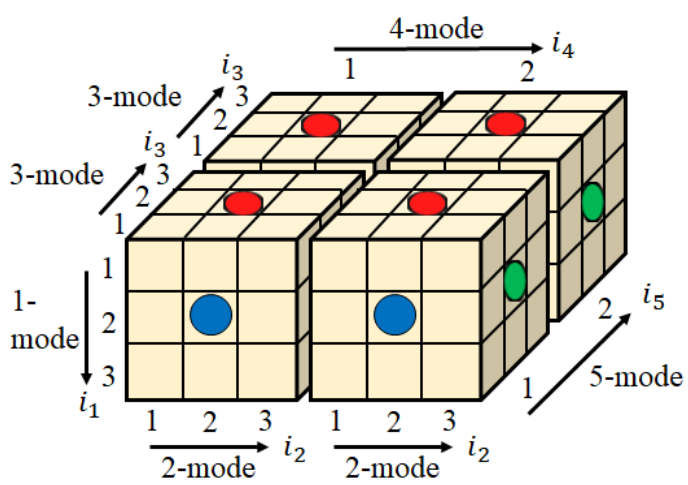

Fig. 5. Fifth-order tensor representation of the 3D puzzle.

[ (Algorithm 3) Map creation of 3D puzzle ] 
Input: A fifth-order tensor $\mathcal{A}$ of size $3 \times 3 \times 3 \times 2 \times 2$

Output: A map matrix $\boldsymbol{A}_{\text {map }}$ of 3D puzzle

(Step 1) Extract the sub-third-order tensor $\boldsymbol{A}_{i_{4}, i_{5}}=\left(a_{* * * i_{4} i_{5}}\right)$, $\left(i_{4}, i_{5}=1,2\right)$ from $\mathcal{A}$, where $\left(a_{* * * i_{4} i_{5}}\right)$ is a third-order tensor with indices $i_{4}$ and $i_{5}$ fixed and the first to third subscripts $i_{1}, i_{2}, i_{3}=1,2,3$.

(Step 2) Apply Algorithm 2 to $\boldsymbol{A}_{i_{4}, i_{5}}, \quad\left(i_{4}, i_{5}=1,2\right)$ obtained in Step 1 to compute the 1-mode matrix unfolding $\boldsymbol{A}_{i_{4}, i_{5}(1)}, \quad\left(i_{4}, i_{5}=1,2\right)$.

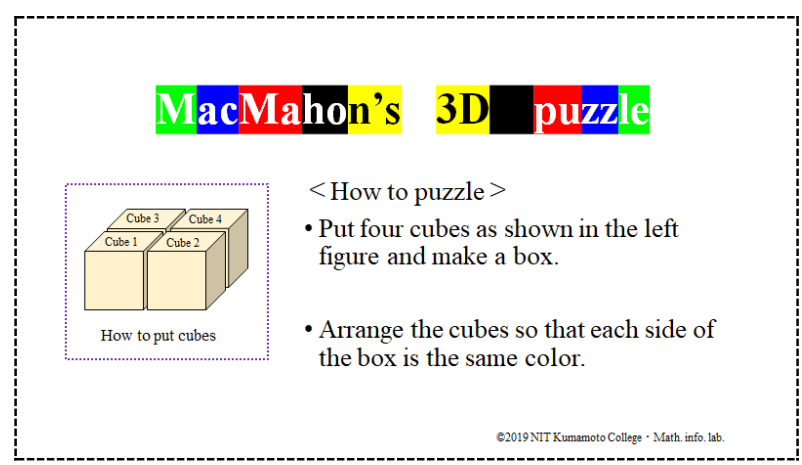

(a) Title screen of the $3 \mathrm{D}$ puzzle
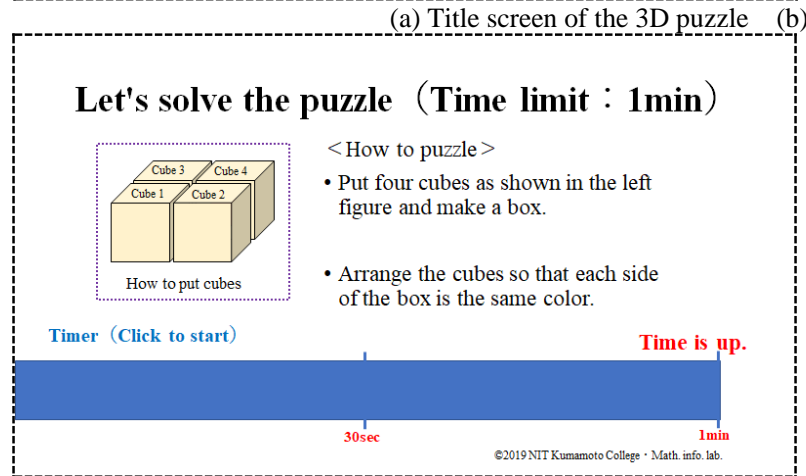

(c) Question screen to solve the puzzle within the time limit (d) Screen of a puzzle map for checking answers

Fig. 6. Example of display screen of developed teaching tool

\section{Teaching ToOl Using Map of 3D PuZZle}

\section{A. Developed Teaching Tool and Its Usage}

A teaching tool for learning the concept and procedure of the $n$-mode matrix unfolding and folding (inverse operation of the unfolding) of HOSVD algorithm was developed using MS PowerPoint application. Fig. 6 shows the example of display screen of these teaching tool. The following (i) to (iv) show the procedure for using the tool.

1) The title screen shown in Fig. 6(a) appears on the display. Next, how to solve the puzzle is explained.

2) Fig. 6(b) is displayed to explain how to read the map. Users practice placing each cube as shown in Fig. 1, while watching the map. Fig. 7 shows this situation. The map in Fig. 6(b) is created by adding color information and position information (that is, Front/Back, Top/Under, and Left/Right.) to the elements having the color values of the map matrix $\boldsymbol{A}_{\text {map }}$ in (4).

3) The question screen shown in Fig. 6(c) is displayed, and the users solve the puzzle within one-minute. When the mouse is clicked, a start tone sounds and the timer works.
(Step 3) The map matrix $\boldsymbol{A}_{\text {map }}$ is constructed by arranging $\boldsymbol{A}_{i_{4}, i_{5}(1)}, \quad\left(i_{4}, i_{5}=1,2\right)$ obtained in Step 2 as shown in the following equation:

$$
A_{\text {map }}=\left(\begin{array}{ll}
A_{1,2(1)} & A_{2,2(1)} \\
A_{1,1(1)} & A_{2,1(1)}
\end{array}\right) .
$$

(Step 4) Return $\boldsymbol{A}_{\text {map }}$.

(End of Algorithm)

In (4), $\boldsymbol{A}_{1,1(1)}, \boldsymbol{A}_{2,1(1)}, \boldsymbol{A}_{1,2(1)}$, and $\boldsymbol{A}_{2,2(1)}$ are partial map matrices for the cubes $1,2,3$, and 4, respectively.

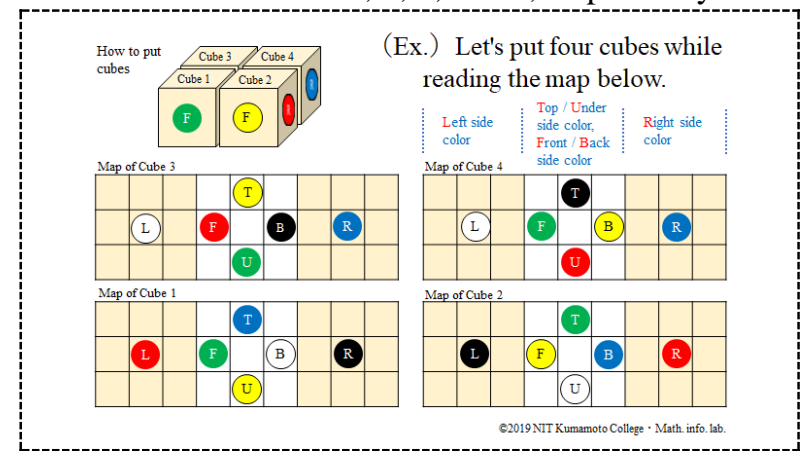

\section{Screen of a puzzle map for exercises}

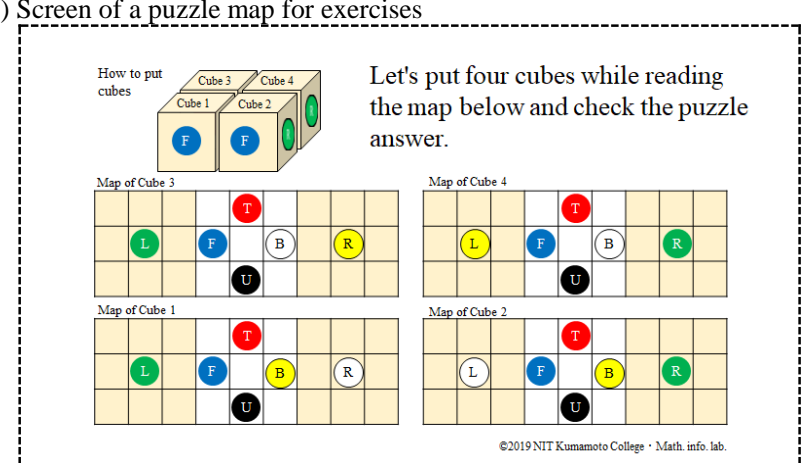

The elapsed time is indicated by a blue bar extending from left to right, as shown at the bottom of Fig. 6(c).

4) Finally, by displaying Fig. 6(d), the user sees an example of the answer of this puzzle. As same as the case of Fig. 6(b), the user actually puts each cube while looking at the answer map.

Because the map of each cube of these teaching tool is created from the 1-mode matrix unfolding, we consider that it is possible to learn the matrix unfolding by repeatedly referencing the map and to learn the folding by actually placing the cubes according to the map.

\section{B. Results of Using This Teaching Tool}

This teaching tool was used by junior high school students and our college students who visited the Campus Open Day held at our college in August 2019. There were 30 users (including 22 junior high school students, 8 our college students and adults). As described in the previous subsection, users were asked to answer simple questions after using the teaching tool. Fig. 8 summarizes the contents of the questions and the results.

Question 1 asks the level of difficulty of the puzzle. 
Although $70 \%$ of users felt that the puzzle was "hard", 16 out of 30 people, more than half of the total, could solve the puzzle within the time limit for the question in Fig. 6(c).

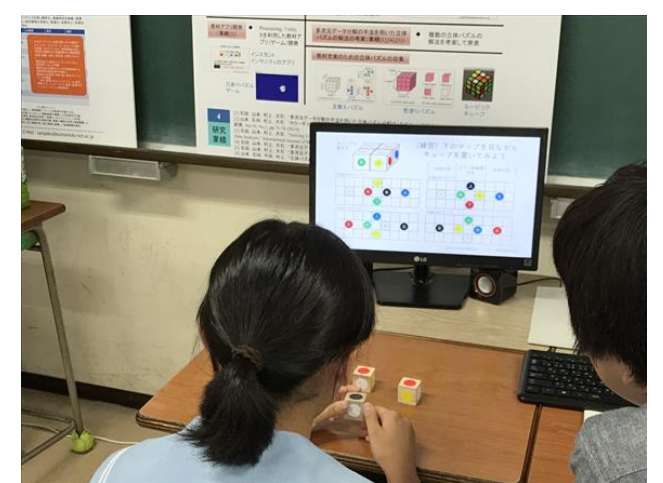

Fig. 7. Scene of exercise of how to read the map.

(Q1) About the difficulty of the puzzle

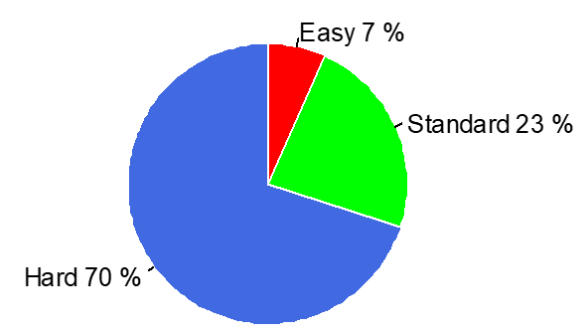

Question 2 is about the readability of the map. The total of "easy to read" and "standard" was about $77 \%$, so we found that the map of this teaching tool is generally easy to read. Also, by checking the map in Fig. 6(d), all users were able to confirm the answer to the puzzle, so it seems that the most users understood the map.

Fig. 9 shows a cumulative relative frequency distribution of the time required for the users to check the answer of the puzzle by placing the cubes as shown in the answer map in Fig. 6(d). The average time required for the confirmation was approximately 63 seconds. We found that about $57 \%$ of users confirmed the answer by this average time, and that about $70 \%$ of them in 80 seconds and about $93 \%$ in 100 seconds completed the confirmation.

\section{(Q2) About the readability of the puzzle map}

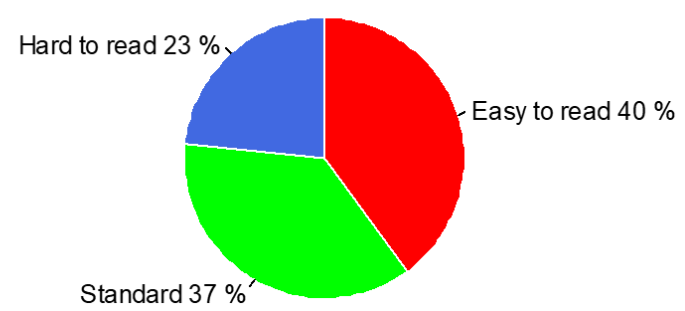

Fig. 8. Summary of questions and answers after use the tool.

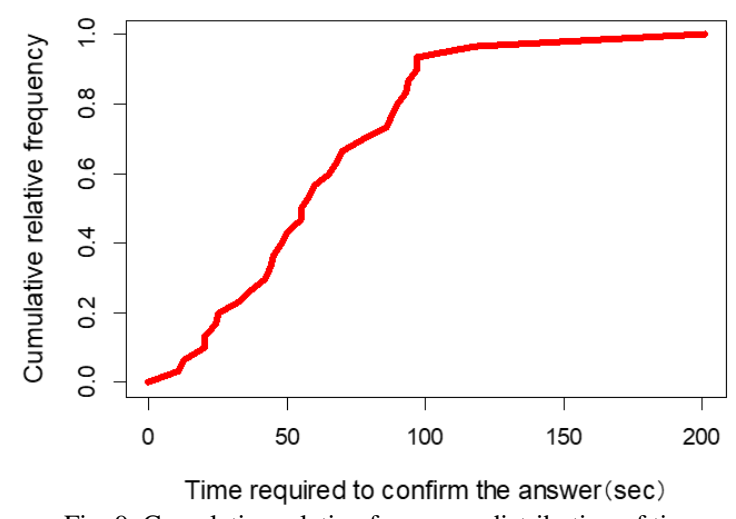

Fig. 9. Cumulative relative frequency distribution of time required to confirm the answer.

\section{CONCLUSIONS}

In this paper, we show a new 3D puzzle created using some of MacMahon's coloured cubes for teaching tensor decomposition. This puzzle is not as difficult as the Instant Insanity and the Rubik's cube, so it can be solved almost within the time limit. By applying the mode matrix unfolding of HOSVD, the puzzle was expressed as a 2D map. Our developed teaching tool allow users to learn matrix unfolding and folding in the calculation process of HOSVD by solving this $3 \mathrm{D}$ puzzle while referring the map.

The developed teaching tool was used by our college students and others at the Campus Open Day to investigate the level of difficulty of the puzzle and the readability of the map. As a result, there were many answers that the difficulty level was high, but it was confirmed that more than half of users can solve the puzzle within the time limit. In addition, we saw that the map is generally easy to read and that almost everyone understood how to read it. From these results, the learners of the concept of matrix unfolding and folding can be understood by using this teaching tool. Our future work is to use this teaching tool in class at our college and to verify that this tool is effective in learning the concept and calculation process of HOSVD.

\section{CONFLICT OF INTEREST}

The authors declare no conflict of interest.

\section{AUTHOR CONTRIBUTIONS}

Naoki Yamamoto conducted the research and wrote the paper; Akio Ishida took responsibility in the construction of the manuscript; Nobuhiro Oishi reviewed the article before submission for its intellectual contents; Jun Murakami organized and supervised the research; all authors discussed progress and results of the research at any time and had approved the final version.

\section{REFERENCES}

[1] A. Cichoki, "Era of big data processing: A new approach via tensor networks and tensor decomposition," arXiv Preprint, arXiv:1403.2048, 2014.

[2] L. Kuang, F. Hao, L. T. Yang, M. Lin, C. Luo, and G. Min, "A tensor-based approach for big data representation and dimensionality reduction," IEEE Transactions on Emerging Topics in Computing, vol. 2, no. 3, pp. 280-291, 2014.

[3] L. Lathauwer, "A survey of tensor methods," in Proc. IEEE International Symposium on Circuits and Systems, pp. 2773-2776, 2009.

[4] A. Ishida, K. Kawakami, D. Furushima, N. Yamamoto, and J. Murakami, "Analysis of relationships between amount of physical activity of patients in rehabilitation and their ADL scores using 
multidimensional PCA," Advances in Intelligent Systems and Computing, vol. 690, pp. 147-158, 2017.

[5] A. Ishida, N. Yamamoto, J. Murakami, and N. Oishi, "Solving 3-D puzzles using tensor decomposition and application to education of multidimensional data analysis," International Journal of Machine Learning and Computing, vol. 8, no. 5, pp. 447-453, 2018.

[6] N. Yamamoto, J. Murakami, and A. Ishida, "Using 3D puzzles to help understand tensor decomposition programming (in Japanese)," The Proceedings of the Annual Software Symposium (SS2019), pp. 114-123, 2019.

[7] J. Köller, "MacMahon's coloured cubes," Mathematische Basteleien, 2002.

[8] L. D. Lathauwer, B. D. Moor, and J. Vandewalle, "A multilinear singular value decomposition," SIAM Journal on Matrix Analysis and Applications, vol. 21, no. 4, pp. 1253-1278, 2000.

[9] E. Larsson and G. Bergqvist, "The higher-order singular value decomposition: Theory and an application," IEEE Signal Processing Magazine, vol. 27, no. 3, pp. 151-154, 2010.

[10] W. Austin, G. Ballard, and T. G. Kolda, "Parallel tensor compression for large-scale scientific data," in Proc. the 30th IEEE International Parallel and Distributed Processing Symposium, pp. 912-922, 2016.

Copyright $(\odot) 2020$ by the authors. This is an open access article distributed under the Creative Commons Attribution License which permits unrestricted use, distribution, and reproduction in any medium, provided the original work is properly cited (CC BY 4.0).

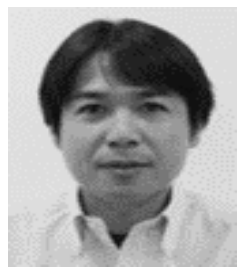

Naoki Yamamoto received the Ph.D. in engineering from Kyushu Institute of Technology, Japan, in 2001 $\mathrm{He}$ is currently a professor of the Department of Human-Oriented Information Systems Engineering, Kumamoto College, National Institute of Technology, Japan. His research interests are in the area of multidimensional data analysis and numerical calculation. $\mathrm{He}$ is a member of the Institute of Electronics, Information and Communication Engineers (IEICE) and Kyushu Society for Engineering Education (KSEE).

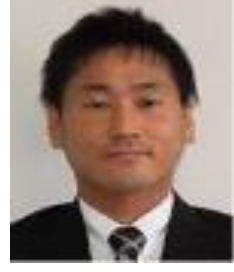

Akio Ishida received the M.S. and Ph.D. in science from Kumamoto University, Japan, in 2010 and 2014. He is currently an assistant professor of the Faculty of Liberal Arts, Kumamoto College, National Institute of Technology, Japan. His research interests are in the area of multi-dimensional data analysis and numerical calculation.

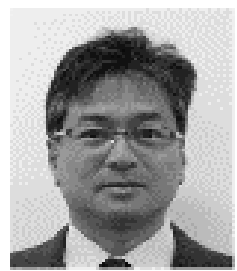

Nobuhiro Oishi received the M.E. from Toyohash University of Technology, Japan, in 1988 and Ph. D. in engineering from Kyushu Institute of Technology, Japan, in 2004. He is currently a professor and the dean of the Department of Information, Communication and Electronic Engineering, Kumamoto College, National Institute of Technology, Japan. His research area of interests includes numerical calculation, statistical analysis, and causal analysis of multidimensional data. He is a member of the Physical Society of Japan (JPS) and the Japan Society of Applied Physics (JSAP).

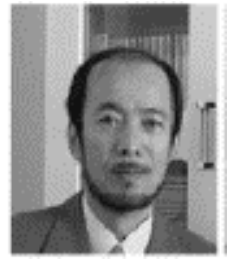

Jun Murakami received the $\mathrm{Ph} . \mathrm{D}$. in engineering from Toyohashi University of Technology, Japan, in 2000. He is currently a professor of the Department of Human-Oriented Information Systems Engineering, the Director of Library, Kumamoto College, National Institute of Technology, Japan. His research area of interests includes statistical analysis, numerical calculation, and digital signal processing. He is a member of the Institute of Electronics, Information and Communication Engineers (IEICE), the Information Processing Society of Japan (IPSJ), and the Human Interface Society Japan (HISJ). 